\title{
CAPITULO 77
}

\section{PREVALÊNCIA DA UTILIZAÇÃO DO DIQUE DE BORRACHA EM PROCEDIMENTO ENDODÔNTICO: REVISÃO INTEGRATIVA}

DOI 10.4322/978-65-995353-2-1.c77

\section{$\underline{\text { Zildenilson da Silva Sousa }}{ }^{1}$, Gilmara Régia da Rocha Lessa Rodrigues ${ }^{2}$, Jamerson} Rodrigues de Sousa ${ }^{3}$, José Auberlanio Lima Rodrigues ${ }^{4}$, Luiza Alana Lopes de Sousa Sampaio $^{5}$, Ana Clara Araújo Fernandes ${ }^{6}$, Claudia Santos de Araújo ${ }^{7}$, Isabela Leal de Moura $^{8}$, Maria da Conceição Freitas da Costa9 ${ }^{9}$, Maria Galba das Neves ${ }^{10}$

${ }^{1}$ Centro Universitário Maurício de Nassau Fortaleza, (zildenilsonsilva@gmail.com) ${ }^{2}$ Centro Universitário Maurício de Nassau Fortaleza, (gilmararegia.gl@gmail.com) ${ }^{3}$ Faculdade de Ensino e Cultura do Ceará, (jamersonr13@gmail.com)

${ }^{4}$ Faculdade de Ensino e Cultura do Ceará, (auberlanio@ hotmail.com) ${ }^{5}$ Centro Universitário Maurício de Nassau Fortaleza, (luiza.alana1906@gmail.com) ${ }^{6}$ Centro Universitário Maurício de Nassau Fortaleza, (clara.a.fernandes@hotmail.com) ${ }^{7}$ Centro Universitário Maurício de Nassau Fortaleza, (claudiasaraujo77@hotmail.com) ${ }^{8}$ Centro Universitário Maurício de Nassau Fortaleza, (isabelaleal1312@gmail.com) ${ }^{9}$ Centro Universitário Maurício de Nassau Fortaleza, (mariafreitas851@gmail.com) ${ }^{10}$ Centro Universitário Maurício de Nassau Fortaleza, (palomagalba395@gmail.com)

\section{RESUMO}

Objetivo: Descrever, por meio de uma revisão integrativa de literatura, a prevalência do uso da barragem de borracha por cirurgiões-dentistas em procedimentos endodônticos. Método: Os descritores "dentistry", "rubber dams", "root canal therapy" e "endodontic treatment" foram aplicados no Sistema Online de Busca e Análise de Literatura Médica (MEDLINE) através da PubMed, além da Biblioteca Virtual em Saúde (BVS) e Science Direct e posteriormente interligados entre sí através dos operadores booleanos "AND/OR" em um período de 10 anos (2011 a 2021). Encontrou-se um total de 522 estudos que após leitura apenas 10 foram incluídos nesta revisão através dos critérios de elegibilidade adotados. Resultados: Observou-se que essa etapa de preparo no tratamento endodôntico é ignorada em alguns países devido ao ambiente de trabalho, preferência dos pacientes, além da redução do tempo clínico, principalmente por cirurgiões-dentistas clínicos gerais. Considerações Finais: É fundamental mais estudos neste campo visto a escassez de dados na literatura, para que assim seja possível fornecer mais informações que promovam o uso deste material por profissionais da odontologia, principalmente na endodontia. 
Palavras-chave: Endodontia; Diques de Borracha; Assistência Odontológica.

Área Temática: Ciências da Saúde.

E-mail do autor principal: zildenilsonsilva@gmail.com

\section{INTRODUÇÃO}

O dique de borracha ou barragem de borracha é um material que tem sido utilizado no atendimento odontológico durante muitos anos. Seu uso é bem descrito em muitos livros didáticos neste campo da ciências da saúde. (ANABTAWI et al., 2013). Esse tipo de material possui diversas vantagens para diversos tipos de especialidades odontológicas visto seu contexto de biossegurança na saúde (CHIANG et al., 2012).

Sua aplicabilidade consiste basicamente em isolar um determinado campo operatório de trabalho do restante da boca através de uma camada bem fina de borracha, com a finalidade de proteger pacientes de uma forma eficaz contra possíveis problemas secundários, como a deglutição de instrumentais. Além disso, facilita a lavagem do campo de trabalho, evitando assim a contaminação salivar daquela área, permitindo ainda a preparação de um campo de trabalho asséptico e de fácil visualização (MADARATI et al., 2016)

Nos últimos anos, essa técnica vem ganhando cada vez mais reconhecimento por parte de dentistas, sendo considerada pelas autoridades em saúde como um procedimento importante para o controle de infecções na odontologia, principalmente durante o tratamento endodôntico (ZOU et al., 2016). Diante disso, o cirurgião-dentista deve conhecer os tratamentos endodônticos mais adequados e como conduzi-los de maneira correta com base na utilização desta peça (FAYE et al., 2017; MASLAMANI e MITRA, 2018).

Todavia, observa-se na literatura que alguns estudos realizados por meio de questionário que há uma frequência alternada do uso desse dique de borracha, o que sugere por pesquisadores que a realização de estudos em diferentes países pode demonstrar diferenças nas práticas e preferências entre os profissionais (MADARATI et al., 2016).

Com base nisso, o conhecimento sobre as atitudes que os dentistas de modo geral possui sobre o uso de barragens de borracha e outros métodos de isolamento ainda precisam ser avaliadas visando entender melhor as causas e a prevalência de fatores que podem influenciar na utilização ou não deste material (GILBERT et al., 2015).

Diante disso, o objetivo do presente estudo foi relatar, por meio de uma revisão integrativa da literatura, a prevalência do uso da barragem de borrada por cirurgiões-dentistas na prática clínica visando identificar os fatores que podem influenciar na adesão ou não do

E - book Tripé do Ensino Superior: Ensino, Pesquisa e Extensão 
material.

\section{REVISÃO DE LITERATURA}

\section{1 BARRAGEM DE BORRACHA E SUA APLICABILIDADE CLÍNICA}

A técnica de isolamento com dique de borracha é um metodologia utilizada na odontologia visando proteger o campo operatório do restante da boca, agindo como uma barreira protetora. De acordo com dados presentes na literatura científica, esse material pode proteger pacientes e dentistas de uma forma eficaz (ZOU et al., 2016). Portanto, deve ser vigorosamente promovido e aplicado na prática clínica (JINGXIAN et al., 2020).

É considerado o padrão ouro no que se refere ao controle de microorganismos na cavidade oral, uma vez que um grupo de pacientes avaliados em tratamento sem o uso de barragens de borracha seria uma prática antiética (AHMAD et al., 2014). Além disso, o uso de rotina da barragem de borracha não deve exigir uma investigação científica para persuadir as atitudes de dentistas de modo geral sobre a necessidade fundamental desta ferramenta durante procedimentos odontológicos (GILBERT et al., 2015; AHMAD et al., 2014).

Estudos sugerem ainda que a prevalência do uso de barragens de borracha por dentistas em diferentes países tende a reduzir consideravelmente após a graduação, uma vez que a utilização do material nas faculdades de odontologia é uma prática obrigatória (ZOU et al., 2016; AHMAD et al., 2014). A causa mais comum pode ser descrita pelo fato de dentistas recém formados podem não estar familiarizados com a gestão lucrativa de um consultório particular, além de fato de estarem fora das regras estabelecidas no ambiente educacional (JINGXIAN et al., 2020; AHMAD et al., 2014).

\subsection{ISOLAMENTO NA ENDODONTIA: EFICÁCIA NO CONTROLE DE MICROORGANISMOS}

A utilização da barragem de borracha pode reduzir o potencial de deglutição de materiais ou objetos usados durante o tratamento de canal, bem como pedaços da estrutura do dentária ou tecido necrótico desalojado durante a preparação do acesso de canal (ANABTAWI et al., 2013; CHIANG et al., 2012).

Diversas técnicas de isolamento podem ser aplicadas na odontologia visando obter um campo operatório estéril. Uma das possíveis explicações possíveis para a baixa utilização de dique de borracha é que os dentistas podem utilizar de uma endodontia rotativa ao fazer o tratamento de canal radicular (ANABTAWI et al., 2013). Esse método funciona com os arquivos fixados na própria peça de mão do profissional, apesar dessa circunstância diminuir a 
probabilidade de um paciente aspirar ou ingerir uma lima, as outras razões para usar uma barragem de borracha não são evitadas (ANABTAWI et al., 2013; AHMAD et al., 2014).

Além da comunicação e do treinamento técnico para profissionais atuantes nesta área da odontologia, os benefícios do uso de barragens de borracha devem ser enfatizados e o uso rotineiro deve ser incentivado (JINGXIAN et al., 2020), uma vez que a turbina de ar resulta na formação de aerossóis e gotículas que geralmente estão contaminadas com o sangue (AHMAD et al., 2009).

\section{MÉTODO}

\subsection{ESTRATÉGIA DE BUSCA}

Trata-se de uma revisão integrativa da literatura realizada entre os meses de julho e agosto de 2021. Para a sua idealização, seguiu-se os 6 passos descritores por Souza, Silva e Carvalho (2010): Elaboração da pergunta norte da pesquisa, busca na literatura de dados relacionados a temática, coleta de informações, análise minuciosa dos estudos selecionados após os critérios de elegibilidade adotados, discussão dos resultados obtidos através da busca literária e apresentação final da revisão.

Nesse sentido, para que fosse possível guiar a busca, realizou o seguinte questionamento: o isolamento absoluto em procedimentos endodônticos é comumente utilizado na prática clínica de cirurgiões-dentistas?" Para a elaboração da pergunta norteadora da pesquisa, seguiu-se o acrônimo PICO: População = cirurgiões-dentistas, "dentistry"; Intervenção = utilização do isolamento absoluto na endodontia, "rubber dams", "root canal therapy”; Comparação/Controle = não utilizado; Desfecho do estudo= eficácia no sucesso do tratamento endodôntico, "endodontic treatment".

Após definição, tais descritores cadastrados nos Descritores em Ciências da Saúde (DeCS) e Medical Subject Headings (MeSH) foram interligados através dos operadores booleanos AND/OR e aplicados na base de dados do Sistema Online de Busca e Análise de Literatura Médica (MEDLINE) através da PubMed, além da Biblioteca Virtual em Saúde (BVS) e Science Direct, sendo encontrados posteriormente um total de 522 estudos publicados em um período de 10 anos (2011 a 2021) que após análise dos critérios de elegibilidade adotados foram selecionados apenas 10 artigos, conforme evidenciado na Figura 1.

\subsection{CRITÉRIOS DE ELEGIBILIDADE}

Foram incluídos nesta revisão estudos com desenho de pesquisas transversais descritivos, estudos originais, estudos pilotos e revisões sistemática publicadas entre 2011 a 
2021, sem distinção de idiomas e que avaliavam a prevalência e os fatores que poderiam estar ligados a utilização ou não do dique de borracha em procedimentos endodônticos por cirurgiões-dentistas.

Foram removidos documentos editoriais, livros e capítulos de livro, estudos incompletos, estudos sem resumo na íntegra, duplicados, estudos indisponíveis, inferiores a 2011, anais de evento, teses e dissertações de mestrado e/ou doutorado, relatos de caso e experiência, trabalhos de conclusão de curso (TCC), estudos que tinham o objetivo de relatar a preferência dos pacientes pelo tratamento com ou sem o dique de borracha e estudos que não relacionavam diretamente a análise e prevalência do uso da barragem de borracha na endodontia por cirurgiões-dentistas na prática clínica, mas sim o seu uso na taxa de sobrevivência de dentes com canais radiculares tratados.

Figura 1. Fluxograma do processo metodológico da seleção dos estudos

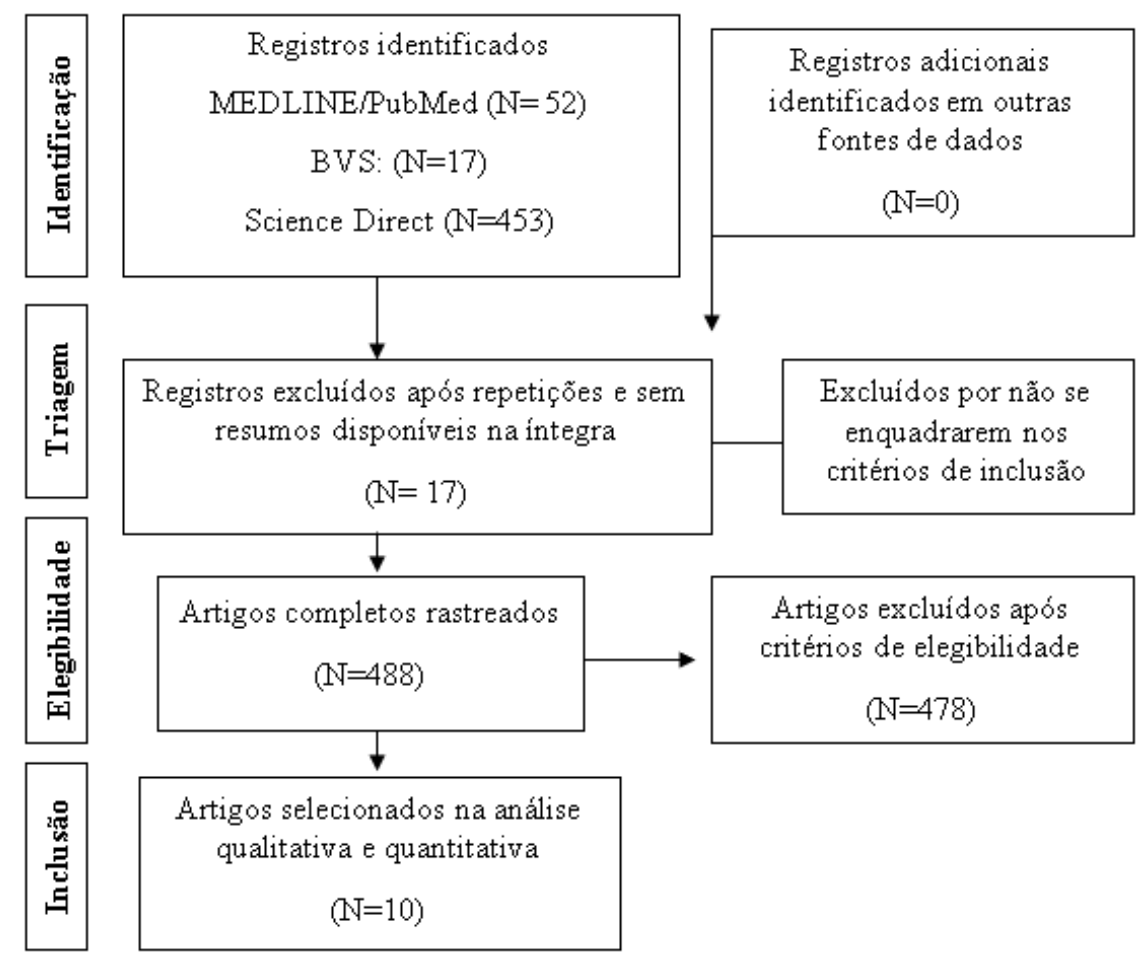

Fonte: Autores, 2021.

\section{RESULTADOS E DISCUSSÃO}

Dentre os achados na literatura, observa-se uma escassez de estudos a longo prazo e com amostras significativas relacionadas à temática em questão. Além disso, observou-se que outros métodos alternativos de isolamento são comumente utilizados por esses profissionais, como a utilização de rolos de algodão e compressas de gazes.

Em 09 estudos, a coleta de dados quantitativos e qualitativos foram através de 
formulários eletrônicos. Assim, nota-se que o isolamento absoluto em procedimentos clínicos na odontologia é uma etapa fundamental visando proteger o paciente de diversos riscos químicos, biológicos e de deglutição de pequenos objetos comumente utilizados por cirurgiõesdentistas.

Contudo, 03 estudos relataram que há uma discrepância do uso desse material na prática clínica entre dentistas clínicos gerais e endodontistas. Dos artigos selecionados, 07 eram estudos transversais descritivos,02 estudos piloto e 01 estudo de revisão. Visando obter um melhor entendimento dos principais achados na literatura, os dados dos 10 estudos selecionados foram destacados na Tabela 2.

Tabela 2. Principais achados na literatura dos estudos incluídos.

\begin{tabular}{|c|c|c|c|}
\hline Autor/ano. & $\begin{array}{c}\text { Desenho do } \\
\text { estudo/País da } \\
\text { pesquisa. }\end{array}$ & $\begin{array}{c}\text { Amostragem } \\
(\mathbf{N})\end{array}$ & Resultados obtidos. \\
\hline $\begin{array}{l}\text { THIESSEN et al., } \\
2020 .\end{array}$ & $\begin{array}{l}\text { Estudo transversal } \\
\text { descritivo. } \\
\text { País: Suíça. }\end{array}$ & $\mathrm{N}=141$. & $\begin{array}{l}86,5 \% \text { dos dentistas relataram fazer uso } \\
\text { rotineiro de barragem de borracha em } \\
\text { tratamentos odontológicos. }\end{array}$ \\
\hline FAYE et al., 2017. & $\begin{array}{l}\text { Estudo transversal } \\
\text { descritivo. } \\
\text { País: Burkina. }\end{array}$ & $\mathrm{N}=33$. & $\begin{array}{l}\text { 9\% dos dentistas não realizavam o uso } \\
\text { de barragem de borracha em } \\
\text { tratamentos endodônticos. }\end{array}$ \\
\hline $\begin{array}{l}\text { MADARATI } \text { et al., } \\
2016 .\end{array}$ & $\begin{array}{l}\text { Estudo piloto. } \\
\text { País: Arábia Saudita. }\end{array}$ & $\mathrm{N}=237$. & $\begin{array}{l}\text { O uso de dique de borracha não é } \\
\text { comumente utilizado por dentistas na } \\
\text { prática odontológica saudita. }\end{array}$ \\
\hline GILBERT et al., 2015. & $\begin{array}{c}\text { Estudo piloto. } \\
\text { País: Não especificado. }\end{array}$ & $\mathrm{N}=1490$ & $\begin{array}{l}\text { Os dentistas gerais apresentam } \\
\text { variações substanciais nas atitudes } \\
\text { sobre o uso de barragens de borracha. }\end{array}$ \\
\hline AHMAD et al., 2014. & $\begin{array}{c}\text { Estudo de revisão. } \\
\text { País: Não especificado. }\end{array}$ & 18 estudos. & $\begin{array}{l}\text { Há uma diferença significativa entre } \\
\text { atitudes de dentistas gerais durante a } \\
\text { graduação e o que praticam após ela. }\end{array}$ \\
\hline $\begin{array}{l}\text { ANABTAWI } \text { et al., } \\
2013 .\end{array}$ & $\begin{array}{l}\text { Estudo transversal } \\
\text { descritivo. } \\
\text { País: Estados Unidos. }\end{array}$ & $\mathrm{N}=729$. & $\begin{array}{l}\text { Nem todos os dentistas usaram um } \\
\text { dique de borracha durante o tratamento } \\
\text { de canais radiculares. }\end{array}$ \\
\hline GUPTA e RAI 2013. & $\begin{array}{l}\text { Estudo transversal } \\
\text { descritivo. } \\
\text { País: Índia. }\end{array}$ & $\mathrm{N}=700$. & $\begin{array}{l}\text { Barragens de borracha eram utilizadas } \\
\text { apenas por } 27 \% \text { dos dentistas da } \\
\text { pesquisa. }\end{array}$ \\
\hline KAPTAN et al., 2012. & $\begin{array}{l}\text { Estudo transversal } \\
\text { descritivo. } \\
\text { País: Turquia. }\end{array}$ & $\mathrm{N}=1400$ & $\begin{array}{l}\text { Quase } 92 \% \text { dos profissionais relataram } \\
\text { que nunca usaram a barragem de } \\
\text { borracha. }\end{array}$ \\
\hline
\end{tabular}




\begin{tabular}{|c|c|c|c|}
\hline $\begin{array}{c}\text { KAPITÁN e } \\
\text { SUSTOVÁ, } 2011 .\end{array}$ & $\begin{array}{l}\text { Estudo transversal } \\
\text { descritivo. } \\
\text { País: República } \\
\text { Tcheca. }\end{array}$ & $\mathrm{N}=449$ & $\begin{array}{l}\text { Frequência reduzida na utilização da } \\
\text { barragem de borracha por dentistas } \\
\text { atuantes na República Tcheca. }\end{array}$ \\
\hline LIN et al., 2011. & $\begin{array}{l}\text { Estudo transversal } \\
\text { descritivo. } \\
\text { País: Taiwan. }\end{array}$ & $\mathrm{N}=1332$ & $\begin{array}{l}\text { A prevalência do uso de barragens de } \\
\text { borracha para tratamento de canal por } \\
\text { dentistas é relativamente baixa. }\end{array}$ \\
\hline
\end{tabular}

Fonte: Autores, 2021.

O uso do dique de borracha durante o tratamento do canal radicular é considerado o padrão de cuidado visto que aumenta a segurança do paciente e otimiza as chances de um tratamento bem-sucedido (GILBERT et al., 2015; GUPTA e RAI, 2013). No entanto, nem todos os dentistas usam, o que gera uma desconexão entre o padrão de atendimento presumido e o que é realmente feito na prática clínica. Além disso, pouco se sabe sobre as atitudes dos dentistas em relação ao uso da barragem de borracha em seus consultórios (GILBERT et al., 2015). Essa condição envolve muitos fatores e deve ser dada a devida atenção (ZOU et al.,2016).

Os resultados mostram que embora a barragem de borracha seja o padrão de atendimento na prática endodôntica, há uma grande diferença entre o que os dentistas são ensinados nas faculdades de odontologia e o que eles praticam ao término dela (AHMAD et al., 2014; KAPITAN e SUSTOVÁ, 2011). Existem poucas evidências científicas para apoiar a aplicação da barragem de borracha; contudo, as diretrizes de prática clínica indicam que é antiético considerar um estudo de coorte para provar o que já é universalmente aceito na prática odontológica (AHMAD et al., 2014; KAPTAN et al., 2012).

Sobre isso, no estudo de Madarati et al. (2016) a taxa de endodontistas que usaram barragem de borracha foi de $84,8 \%$, o que foi significativamente maior que a de dentistas gerais sem especialidade na área $(21,6 \%)$. Observou-se ainda através de seu estudo piloto que a maior proporção não utilizava a barragem de borracha por indisponibilidade do material no local de trabalho (40,5\%), o que fez com que esses profissionais utilizassem meios de isolamento como rolos de algodão no local de tratamento para finalização do procedimento.

Similar aos resultados de Madarati et al. (2016), Lin et al. (2011) observaram que a prevalência do uso de barragens de borracha para tratamento de canal radicular por dentistas do sistema de Seguro Saúde Nacional em Taiwan também demonstrou-se reduzida, em termos estatísticos representou apenas $16,5 \%$ da amostra total coletada pelos autores. Com base nisso, para os autores, o isolamento com dique de borracha em um dente tratado endodonticamente pode fornecer melhor controle de infecção, além de aumentar a proteção do paciente e melhorar

\section{E - book Tripé do Ensino Superior: Ensino, Pesquisa e Extensão}


a eficiência do tratamento. Apesar disso, ainda há uma necessidade de promover seu uso para cada caso de tratamento de canal radicular.

O estudo de Thiessen et al. (2020) difere de Madarati. (2016) e Lin et al. (2011) em termos de aplicabilidade clínica do material. Os autores observaram que a maioria dos dados coletados através de formulários demonstrou que os profissionais realizam cotidianamente o uso da barragem de borracha em procedimentos odontológicos. Apesar dos efeitos positivos, o estudo demonstra escassez de informações em áreas específicas da odontologia visto que não avaliou a aplicabilidade da ferramenta no tratamento endodôntico.

Além do uso de barragem de borracha, outras formas de isolamento também foram relatadas na literatura. Nessa perspectiva, no estudo de Anabtawi et al. (2013), dos 524 dentistas que realizaram tratamento de canal radicular e relataram a porcentagem de tempo em que usam uma barragem de borracha, 204 relataram pelo menos um outro tipo de isolamento, como a utilização de rolos de algodão. Neste estudo, observou-se ainda que o uso de dique de borracha foi universal entre os endodontistas quando levado em comparação a dentistas gerais.

\section{CONSIDERAÇÕES FINAIS}

De acordo com dados presentes na literatura avaliada, a prevalência no uso do isolamento com dique de borracha em procedimentos endodônticos por dentistas é variável, uma vez que o ambiente de trabalho pode ser modificado dependendo de fatores culturais e econômicos de cada país. Dentre as limitações desta revisão, observa-se nos estudos coletados amostras reduzidas, o que sugere novas abordagens na coleta de dados visando um número maior de profissionais participantes. Este estudo pode contribuir para fins informativos a cirurgiões-dentistas sobre a prevalência do uso deste material na endodontia para possíveis estudos na área, visando obter melhores medidas que promovam o seu uso na odontologia, para que assim possa reduzir riscos e promover um tratamento odontológico adequado ao paciente.

\section{REFERÊNCIAS}

AHMAD, I. A. Rubber dam usage for endodontic treatment: a review. International Endodontic Journal, [S.L.], v. 42, n. 11, p. 963-972, 2009.

AHMED, H. et al. Rubber dam application in endodontic practice: an update on critical educational and ethical dilemmas. Australian Dental Journal, [S.L.], v. 59, n. 4, p. 457-463, 2014.

ANABTAWI, M. F. et al. Rubber dam use during root canal treatment. The Journal Of The

E - book Tripé do Ensino Superior: Ensino, Pesquisa e Extensão 
American Dental Association, [S.L.], v. 144, n. 2, p. 179-186, 2013.

CHIANG, C.P et al. Low prevalence of rubber dam usage for root canal treatment in Taiwan. Journal Of The Formosan Medical Association, [S.L.], v. 111, n. 11, p. 660, 2012.

FAYE, B. et al. A Survey of Endodontic Practices among Dentists in Burkina Faso. The Journal Of Contemporary Dental Practice, [S.L.], v. 18, n. 8, p. 641-646, 2017.

GILBERT, G. H et al. Discordance between presumed standard of care and actual clinical practice: the example of rubber dam use during root canal treatment in the national dental practice-based research network. Bmj Open, [S.L.], v. 5, n. 12, p. 79-97, 2015.

GUPTA, R; RAI, R. The Adoption of New Endodontic Technology by Indian Dental Practitioners: a questionnaire survey. Journal Of Clinical And Diagnostic Research, [S.L.], v. 7, n. 11, p. 2610-2614, 2013.

JINGXIAN, F. et al. Application of Quality Control Circle in Promoting the Use of Rubber Dams in the Root Canal Treatment of Primary Teeth. Biomed Research International, [S.L.], v. 2020, p. 1-5, 2020.

KAPTAN, R. F. et al.. An Investigation of Current Endodontic Practice in Turkey. The Scientific World Journal, [S.L.], v. 2012, n.2, p. 1- 6, 2012.

KAPITÁN, M; SUSTOVÁ, Z. The use of rubber dam among Czech dental practitioners. Acta Medica (Hradec Kralove), v.54, n. 4, p.144-148, 2011.

LIN, P. Y. et al. The Effect of Rubber Dam Usage on the Survival Rate of Teeth Receiving Initial Root Canal Treatment: a nationwide population-based study. Journal Of Endodontics, [S.L.], v. 40, n. 11, p. 1733-1737, 2014.

LIN, H.C. et al. Use of Rubber Dams During Root Canal Treatment in Taiwan. Journal Of The Formosan Medical Association, [S.L.], v. 110, n. 6, p. 397-400, 2011.

MASLAMANI, M; MITRA, A. Factors associated with patients' satisfaction of rubber dam use during root canal treatment. Indian Journal Of Dental Research, [S.L.], v. 29, n. 2, p. 144-149, 2018.

MADARATI, A. A. Why dentists don't use rubber dam during endodontics and how to promote its usage? Bmc Oral Health, [S.L.], v. 16, n. 1, p. 16-24, 2016.

MADARATI, A. et al. Dental-Dam for Infection Control and Patient Safety during Clinical Endodontic Treatment: preferences of dental patients. International Journal Of

Environmental Research And Public Health, [S.L.], v. 15, n. 9, 2012.

E - book Tripé do Ensino Superior: Ensino, Pesquisa e Extensão 
SOUZA, M. T; SILVA, M. D; CARVALHO, R. Revisão integrativa: o que é e como fazer. Einstein (São Paulo), São Paulo, v. 8, n. 1, p. 102-106, 2010.

THIESSEN, M. et al. What happened to our former students five to six years after graduation? An endodontic teacher's perspective. Swiss Dental Journal, v. 7, n.8, p. 584-591, 2020.

ZOU, H. et al. An overview on rubber dam application in dental treatments. Yiigle, [S.L.], v. 51, n. 2, p. 119-123, 2016. 